\title{
A comprehensive review on polymeric micelles: a promising drug delivery carrier
}

\begin{abstract}
The main aim of drug delivery systems is to regulate the rate of drug release as per the patient's physiological conditions as well as the progression of the illness or as per the circadian rhythms. To achieve such objectives, the new drug delivery systems have been developed to provide the drug release profile, which is based on each patient's needs. Different researches have been done to create drug delivery carriers, focusing on targeting and delivering hydrophobic drug molecules. This review focuses on Polymeric Micelles as the promising drug delivery carrier due to its high stability, protective property against the harsh gastrointestinal environment.
\end{abstract}

Keywords: polymeric micelles, hydrophobic drug molecule, promising drug delivery carrier
Volume I0 Issue 3 - 202I

\author{
Ankit Shukla,' Shikha Tiwari,, Mahendra \\ Pratap Singh, ${ }^{3}$ Shivjeet Singh, ${ }^{4}$ Mahendra \\ Kumar Singh, ${ }^{5}$ Ajay Kumar ${ }^{5}$ \\ 'Dr. Om Prakash School of Pharmacy, Farrukhabad, Uttar \\ Pradesh, 20956I, India \\ ${ }^{2} \mathrm{Om}$ Sai Vindhya College of Pharmacy, Mirzapur, Uttar Pradesh, \\ 231310 , India \\ ${ }^{3}$ Krishnarpit Institute of Pharmacy, Prayagraj, Uttar Pradesh, \\ 212107, India \\ ${ }^{4}$ Government Mahila Polytechnic, Rasulabad, Prayagraj, Uttar \\ Pradesh, 211013 , India \\ ${ }^{5}$ Government Pharmacy College, BRD Medical College, \\ Gorakhpur, Uttar Pradesh 2730 I3, India
}

Correspondence: Ajay Kumar, Government Pharmacy College, BRD Medical College, Gorakhpur, Uttar Pradesh 2730I3,

Email ajaykumarbp@gmail.com

Received: April 15, 202I | Published: May 04, 202 |

\section{Introduction}

The essential requirement of any drug delivery system is its ability to deliver an active drug molecule at the particular site and at a rate that can be adjusted at every moment as per the progression of disease 1 . The new approaches used in the delivery of drugs from any drug carrier are based on different designed criteria compared to controlled and sustained drugs, which only provide a modified released profile for a prolonged period. ${ }^{1,2}$ From a therapeutic aspect, the discontinuous release of any medicament as a function of specific signals can be profitable in many cases. ${ }^{3}$ This can be utilized in different situations where: a) the drug is volatile in the biological system, and premature delivery of a drug leads to drug degradation before reaching their site of action (in case of therapeutic peptides and proteins); b) The toxicity of the drug causes accumulation of sufficient concentration at the site of action with minimal exposure to the other organs or cells (in case of cancer chemotherapeutics); c) the drug must reach, cellular structure, tissues, cells that are not easily accessible from the general circulation (in case of gene therapy) and the d) the levels of the drug must be according to biorhythms, ${ }^{4}$ as occurs when estrogens, gastric acid inhibitors or medications for heart rhythm disorders or asthma are administered. ${ }^{5}$ The prime aspect represents the most challenging quality for a drug delivery system that warrants the highest smartness level. While delivering the drug at the specific location needs surface modification with a relevant targeting moiety that will enable the carrier molecule to bind to the cells of interest, delivery of a therapeutic dose of a drug required the incorporation of a trigger molecule that will control the amount of drug released at a given time. The trigger may be a stimulus provided externally or could be due to the biological system's internal environment in which the drug delivery carrier is imported. External triggers include electromagnetic radiation, electric or magnetic field, temperature, or ultrasound that could be used to on or off the carrier's drug release. While internal triggers include temperature, change in $\mathrm{pH}$, proteins, ionic environment, carbohydrates, etc. ${ }^{6}$

\section{What is smart drug delivery system?}

The intelligent Drug Delivery carrier or Smart Delivery system has gain importance nowadays as they release the drug from the delivery system as a function of specific stimuli. They are capable of releasing the active drug moiety as per the body's physiological needs. The prime concern for designing an intelligent delivery system is to control the rate of drug release, dosage adjustment as per demand, target specificity, mass transfer, and stability in the biological system. Drug-delivery systems continue to be developed, advanced, refined, and enhanced to provide better treatment options, promising an enhancement in the quality of life and extension of life expectancy. ${ }^{7}$

The intelligent drug delivery system has three functions - sensor, processor, and effector mechanism. The smart drug delivery system identifies the signal caused due to disease with the magnitude of the signal lastly releases the drug from the particular dosage form. The intelligent drug delivery system act in two ways: Open-loop systems, which are also known as pulsatile, which control the drug release by sensing a specific external stimulus like a magnet, ultrasound, and electrical effect. The release rate is independent of the conditions of the biological environment. The intelligent drug delivery also worked on closed-loop or self-regulated systems that directly respond to the changes in the biological medium like changes in the $\mathrm{pH}$, the concentration of some substances or the temperature, which modulates the response activating or modulating the reaction and thus modifies the drug release. ${ }^{8}$

It is excellent to improve different intrinsic properties like Absorption, Distribution, Metabolism, and the elimination properties of a drug molecule by developing various types of drug therapies. Different types of drug delivery carriers to improve the solubility, stability, and efficacy of chemical entities. Thus, it becomes challenging to develop an ideal drug delivery system with high compatibility and minimum cytotoxicity. The category of drugs with low aqueous solubility and newly developed medications with inadequate ADME 
leads to a decrease in the drug molecule's therapeutic index. Thus there is an increase in the advancement of Intelligent drug delivery systems based on the utilization of novel biomacromolecules. The unique approach to developing the delivery system is to release the active moiety at the targeted site, and modification of the rate of release of drug from particular formulation depends on the patients' physiological conditions, progressive illness of the patients, and the circadian rhythms. ${ }^{9}$ The Intelligent drug delivery system has found that they can deliver the active drug molecule at the desired site containing biocompatible polymers. Different types of polymers can be utilized which are sensitive to physiological variables. ${ }^{10}$

\section{Why did polymeric micelles serve as an intelligent drug delivery carrier?}

From the different researches, it has been found that Polymeric Micelles served the Intelligent Drug Delivery System's purpose. They are the result of the association of polymeric chains that are sensitive to stimuli. The polymers are kept together through weak interactions in the polymeric micelles. Polymeric Micelles are nano-sized shell structure formed by amphiphilic block copolymers. From the findings, it has been found that the Polymeric Micelles have gain importance due to the following properties: drugs.

They have the capacity for the solubilization of poorly soluble

I. Small in size.

II. Capable of sustainable delivery of active drug molecules

III. Low molecular weights drugs can be easily administered.

IV. Protect encapsulated material from degradation and metabolism ${ }^{11}$.

V. The nano-sized aggregates of micelles form spontaneously in aqueous solutions and thus increases the half-life of a drug.

The study aims to elaborate information regarding the role of Polymeric micelles for the intelligent delivery of drugs. The polymeric micelles consist of a core-shell structure with a versatile drug-loading hydrophobic core and biocompatible hydrophilic shell and are nanometers in size. Due to the development of different nanotechnology-based methods, polymeric therapeutics has been an emerging aspect of drug delivery and genes. ${ }^{11}$

\section{Formation of polymeric micelles}

Polymeric Micelles are self-assembled core-shell nanostructures formed in an aqueous solution consisting of the amphiphilic block. The creation of micelles in an aqueous solution occurred when the concentration of the block copolymer increases above a particular level named the critical aggregation concentration or critical micelle concentration. At CMC, block copolymers start to associate hydrophobic segments with minimizing the contact with water molecules, leading to the formation of a vesicular or core-shell micellar structure. ${ }^{12}$ Polymeric micelles used for intelligent drug delivery is now a promising study of research in the field of drug delivery and targeting. They are the self-assembled nano-sized colloidal particles containing polymer chains. They can be self-assembled units in any liquid by composing with hydrophobic polymeric segments. Micelles usually have a core-shell structure, in which the core contains either the hydrophobic part of the ionic composition of the nanoparticles containing drug molecule within it., The shell interacts with the solvent and forms the nanoparticles, which is stable in any liquid medium Figure 1. ${ }^{13}$

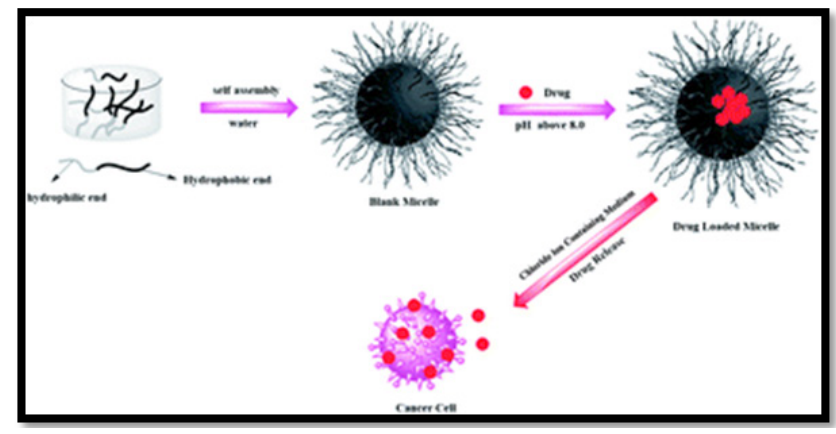

Figure I Formation of Polymeric micelles. ${ }^{13}$

\section{Structure analysis and chemistry of polymeric micelles}

As we are all aware that the amphiphilic molecules or surfactant monomers possess a polar head and a nonpolar tail resulting in changes in physicochemical properties, further results in the formation of Micelles. The significant difference between the micelles of conventional monomers and polymeric micelles is that there usually is a covalent linkage in individual polymeric surfactant molecules within the hydrophobic core that does not allow the dynamic exchange of monomers between free solution and the micellar pseudo-phase. This confers rigidity and stability to the polymeric micelles. The diameter of the polymeric micelle ranges from 10 to $100 \mathrm{~nm}$. The micellar core produces a hydrophobic domain. The polymeric micelles are used to solubilization hydrophobic moieties in the core region through hydrophobic interactions and ionic interactions. Most of the poorly water-soluble drugs can be easily incorporated into the core of polymeric micelles to overcome solubility problems. Solubility enhancement usually is associated with the betterment of oral bioavailability of the hydrophobic drugs. ${ }^{14}$

The inner core of the Polymeric micelles was formed with hydrophobic blocks of the copolymers by hydrophobic interaction. It can also be formed by electrostatic interactions, using charged block copolymers of oppositely charged macromolecules, resulting in the formation of polyion complex (PIC) micelles. As from the previous studies, the micelles are formed when any surfactant molecule is dissolved at the concentration above Critical Micelle Concentration Figure $2 .{ }^{15}$ The micelles are formed due to the occurrence of two types of forces; one is the force of attraction, which causes association of different micelle. The other force that governs the formation of micelles is a repulsive force that prevents the unlimited growth of micelles. ${ }^{16}$ Recent studies have shown that different macromolecular carriers like water-soluble polymers, dendrimers, and polymeric micelles are utilized for smart drug delivery. Polymeric Micelles are most commonly used for the intelligent delivery carrier has advantageous features to provide structural flexibility, multiple functional moieties, a sequestered nano depot with robust stability. ${ }^{17}$

The advantage of utilizing polymeric micelles as carrier systems is that they can undergo dynamic physicochemical changes during drug entrapment and release in molecular form and dissociate between different block copolymers components. ${ }^{17}$ From the studies, it has been reported that Polymeric micelles are also formed by complexation by forming hydrogen bond and are also established by metal ligands coordination bond. The outer shell of Polymeric micelles is formed by the copolymers' hydrophilic blocks, which are used to provide stabilization and direct interaction with the cells. Lengths of the hydrophilic and hydrophobic blocks affect the conformation of polymers in the medium, as hydrophilic blocks of polymer cause it to remain monomeric in water. The micelles are 
formed when the concentration of block copolymers increases Critical Aggregation Concentration. At such a level, the hydrophobic portion of block copolymers starts to associate with minimizing the contact with water molecules, leading to the formation of a vesicular or core-shell micellar structure. ${ }^{18}$ The three most commonly used block copolymer in polymeric micelles classes are characterized by their hydrophobic blocks are poly (L-amino acid)s, poly (propylene oxide), and poly(ester)s.

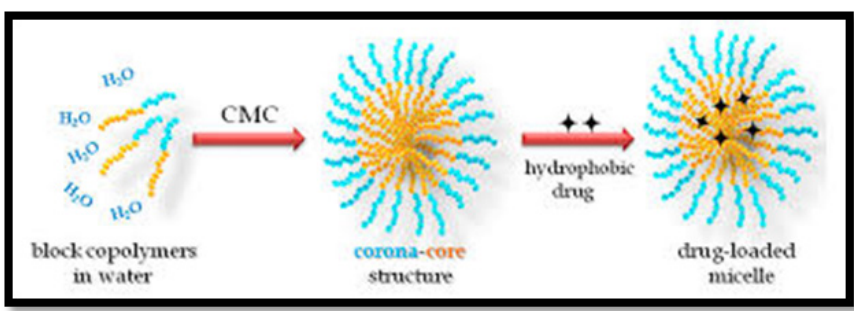

Figure 2 Polymeric Micelles. ${ }^{15}$

\section{Polymeric micelles}

\section{Types of polymeric micelles}

They are classified into three main categories based on intermolecular forces, which regulates the separation of the core segment from the aqueous environment.

I. Micelles are formed by hydrophobic interactions.

II. Poly ion complex micelles formed from electrostatic interactions.

III. Micelles formed from metal complexation. ${ }^{18}$

\section{Conventional polymeric micelles}

Hydrophobic interactions between the core segment and the corona region in the aqueous environment formed these types of micelles. One of the simplest amphiphilic block copolymer, poly(ethylene oxide)b-poly(propylene oxide)-b-poly(ethylene oxide) forms micelles as a result of 16 hydrophobic interactions.

\section{Poly ion complex}

Electrostatic interaction between two oppositely charged moieties, such as poly electrolytes, also leads to polymeric micelles formation. When oppositely charged polymers are added in the medium, they can enter the micelle's corona and give rise to polyionic micelle. Such formed micelles are termed polyion complex micelles (PICMs). The electrostatic forces and the van der Waals force of interaction control the charged micelle coronas' structure and size. The preparation of micelles is carried out in an aqueous medium without the involvement of any organic solvents, thus removing the associated side-effects produced by the residual organic solvents. The core of polyion micelles can entrap many therapeutic agents such as hydrophobic compounds, hydrophilic compounds, metal complexes, and charged macromolecules through electrostatic, hydrophobic, hydrogen bonding interactions, and release them after receiving a suitable trigger Figure 3. ${ }^{19,20}$

\section{Non-covalently connected polymeric micelles}

A novel "block-copolymer-free" technique can also be used for preparing polymeric micelles. Here, polymeric micelles are obtained by self-assemblage of homopolymer, random copolymer, graft copolymer, or oligomer for which interpolymer hydrogen bonding complexation serves as the driving force. Core and shell are noncovalently connected at their homopolymer chain end by specific intermolecular interactions such as H-bonding or metal-ligand interactions in the resultant structures. Hence, these are termed as non covalently connected micelles. ${ }^{20}$

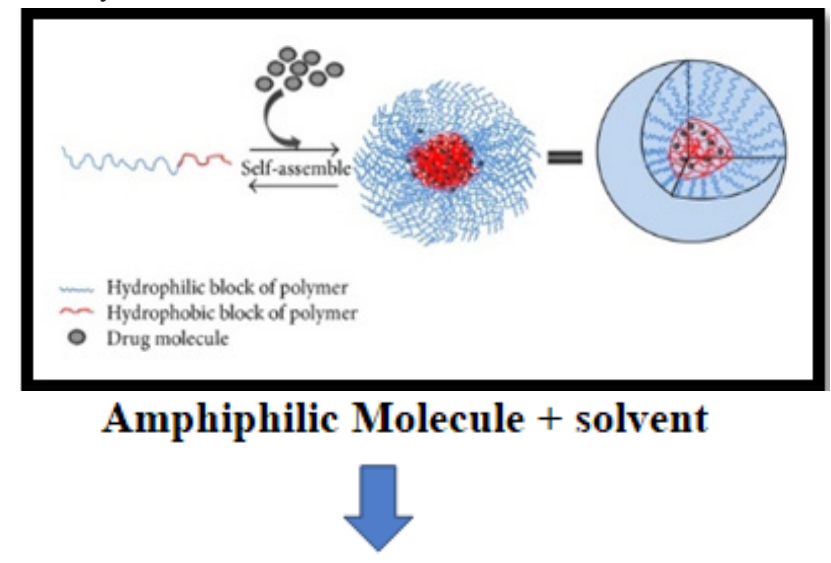

\section{Self Association of Ampiphillic Molecule}

\section{Polymeric Micelles}

Figure 3 Formation of Polymeric Micelles. ${ }^{18}$

Why are polymeric micelles gaining importance in an intelligent drug delivery system?

As per the literature reviews, it has been found that the polymeric micelles have been gaining importance as Smart drug delivery Carrier. Can utilize them as:

\section{Polymeric micelles can be used for enhancement of bioavailability}

The polymeric micelles can protect the loaded drug from the harsh environment of the GI tract, and they can release the drug in the controlled form at the targeted site; the polymeric micelles can prolong the residence time of the drug by mucoadhesion. ${ }^{21}$ Several modifications are done to the polymeric micelles, like particle size, making them easier to cross the intestinal epithelium. ${ }^{22,23}$ Thus polymeric micelles can be fabricated to different carrier systems to enhance bioavailability.

\section{Mucoadhesive polymeric micelles}

The mucosal retention can be used to increase the transit time in the GI tract resulting in the extended time window for the payload release. The mucoadhesive polymeric micelles swell and fill the mucous membranes crevices, contributing to the active surface area in contact with the intestinal mucosa and yielding a high local concentration of the drug. ${ }^{24}$ The muco adhesion process can also localize the polymeric micelles at the target site. The process can be achieved by building either nonspecific interactions with the mucosal surface such as covalent bonds or specific interactions by functionalizing polymers with targeting ligands like lectins or reactive thiols. ${ }^{25}$ The polymers such as cross-linked polyacrylic acids (PAA), carboxy polymethylene, carboxymethyl cellulose, alginate, chitosan(CS), and their derivatives are commonly accepted as mucoadhesive and safe polymers Figure $4 .^{25}$

\section{pH-sensitive polymeric micelles}

The utilization of polyacids or polybases can be used as building blocks of polymeric micelles to provide a ph sensitivity of drug release 
as the polymeric micelles are formed at the $\mathrm{pH}$ above the $\mathrm{pKa}$ of the protonable group while the hydrophobic part remains uncharged. As the $\mathrm{pH}$ decreases below the $\mathrm{pKa}$, the polymer's ionization causes increased hydrophilicity and electrostatic repulsions of the polymers, leading to the destabilization of the micelles and controlled drug release Figure $5 .{ }^{26}$ From the researches, it has been found that the $\mathrm{pH}-$ sensitive polymeric micelles have great potential to act as synthetic vectors for systemic gene delivery. The DNA molecule interacts with the copolymer's amino groups, which forms a complex that is included inside the micelle (polyplex micelle), which protects it from enzymatic degradation. For example, micelles of poly (ethylene glycol)-b-poly [(3-morpholinopropyl) aspartamide]-b-poly(l-lysine) (PEG-b-PMPA-b-PLL) combine the buffering capacity of PMPA with the excellent aptitude to condense DNA of PLL, resulting in a high transfection efficiency. ${ }^{26}$

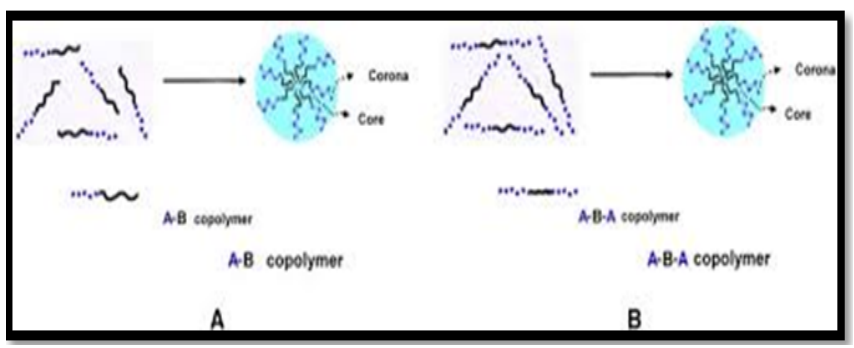

Figure 4 Formation of Mucoadhesive Polymeric Micelles. ${ }^{25}$

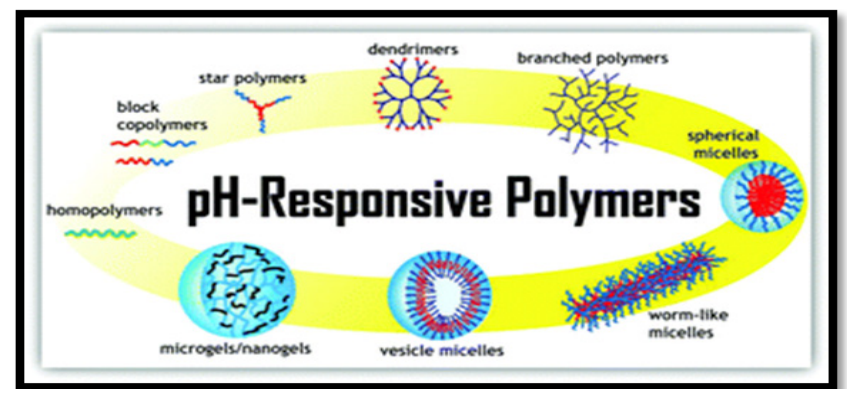

Figure 5 pH-Responsive Polymeric Micelles. ${ }^{26}$

\section{Temperature-sensitive polymers}

It is used to prepare intelligent systems that are hydrophilic below their critical temperature of dissolution. When the temperature is above Lower Critical Solution Temperature, the polymer becomes hydrophobic, and its conformation changes from soluble to insoluble state. Amphiphilic copolymers containingpoly(N-isopropyl acrylamide)one of its derivatives can be used to obtain temperaturesensitive micelles. ${ }^{27}$ Above this temperature, the micelle destabilizes, and the drug is released. The most common example is Doxorubicin, the micelles of this copolymer slowly release the drug at $37^{\circ} \mathrm{C}$, but the release becomes faster when the temperature rises to $42^{\circ} \mathrm{C} 28$. The micelles could be useful for developing intelligent systems that release the drug when hyperthermia occurs either at a systemic level or a specific region. The release could also be triggered by an external source of heat applied to a delimited area of the body Figure $6 .{ }^{29}$

\section{Light sensitive polymeric micelles}

Light as a triggering agent by utilizing polymeric micelles enables the localized release of drugs and minimizes the effect on adjacent tissues. Ultraviolet light can be used for treatments applied to the skin or the mucous and generally used in photodynamic therapy. In the polymeric micelles with photoactive groups when exposed to the light, copolymers undergo reversible alterations of the hydrophilic-lipophilic balance have been assayed as components of light-sensitive micelles. The reviews have found that light-sensitive polymer micelles, nano-, and microgels prepared with amphiphilic or double-hydrophilic block copolymers containing coumarin moieties. From the reviews, it has been found that with amphiphilic BCPs, by incorporating coumarin chromophores into shell-forming block, photo-controlled stabilization and de-stabilization of micelles was achieved by reversible photo-crosslinking of polymer chains based on the reversible photodimerization of coumarin groups and photocleavage of cyclobutane bridges. ${ }^{30}$

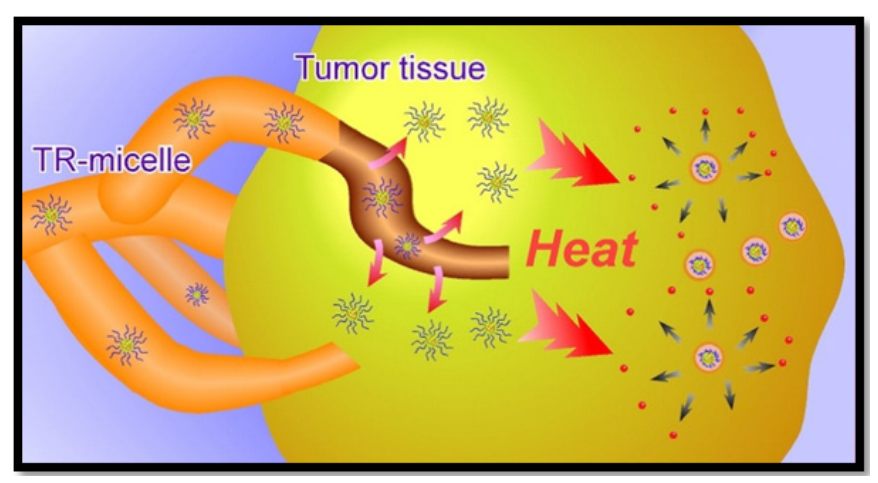

Figure 6 Temperature-sensitive polymeric micelle carriers accumulate at tumor tissues and enhance drug release and cellular uptake by the exogenous administration of heat. ${ }^{29}$

\section{Ultrasound sensitive polymeric micelles}

Polymeric micelles can be destabilized by applying ultrasounds for pulsatile drug delivery in tumors30. Once iv injected, polymeric micelles accumulate in tumor tissues via the EPR effect. The ultrasounds should be used when maximum micelle accumulation in the tumor is reached; the waiting period depends on the kinetics of the micellar system's distribution. The amount of drug released can be modulated by controlling the frequency, power density, pulse length, and inter-pulse intervals. ${ }^{30}$ Thus, polymeric micelles have been gaining importance in different pharmaceutical aspects. The review has collected how the mixed polymeric micelles can be characterized as per their different physiological properties.

\section{Evaluation of polymeric micelles}

\section{Determination of solubilising efficiency}

The solubilisation ability of polymeric micelles was compared with its enhancement capacity as compared to pure drug form. To evaluate the enhancement capacity, the pure drug and polymeric micelles were suspended in distilled water followed by mechanical agitation, and centrifugation was carried out 24 hours at a very high speed. After that, the filtrate obtained from the drug was analyzed with the help of a UV Spectrophotometer. ${ }^{31}$

\section{Determination of particle size, polydispersity and zeta potential}

The Particle size, polydispersity, and hydrodynamic diameter were measured by dynamic light scattering (DLS). The multi-model size distribution of polymeric micelles is determined by atomic force microscopic techniques. The technique can be also be used to differentiate between single, fused, and aggregate particles. ${ }^{32}$

\section{Determination of encapsulation efficiency of polymeric micelles}

The weighed amount of drug-loaded in the polymeric micelles was dissolved in alcoholic solutions, and micelles are broken with the sonication method's help. UV Spectrophotometer analyses the polymeric micelles. 
The formula determines the encapsulation efficiency:

Encapsulation capacity $=$ actual weight of drug/ theoretical weight of drug $* 100$

\section{In vitro release studies release study}

The rate of drug release from the micelle formulation in vitro is checked by a dialysis method. The dialysis was carried out at $37^{\circ} \mathrm{C}$ using Spectra/Pordialysis membrane by maintaining appropriate sink conditions. The micelles loaded with the drug was placed in a dialysis bag. The end sealed dialysis bag is immersed into $100 \mathrm{~mL}$ of PBS (pH 7.4) at $37^{\circ} \mathrm{C}$ with high-speed stirring. ${ }^{32}$

\section{Conclusion}

From the different studies carried out about polymeric micelles, it has been found that they are promising drug delivery carrier for the poorly water-soluble drug as it enhances the oral drug bioavailability due to it special stability arrangements as they belong to supramolecular core-shell-type assemblies. By the utilization of polymeric micelles, can also minimize repeated dosing during chronic conditions. Thus to achieve excellent oral absorption of poorly watersoluble drugs, the loaded drug should be protected from the harsh gastrointestinal environment and release in a controlled manner at the target sites. In this review article, we have illustrated the potential of polymeric micelles for delivery of poorly water-soluble drugs, especially in the areas of oral delivery. A required field that verifies the effectiveness of micellar drug carriers is controlling the location and time over which drug release occurs. The $\mathrm{pH}$-sensitive polymeric micelles, light-sensitive, ultrasound sensitive, temperature-sensitive polymeric micelles have emerged as fascinating drug carriers that can be easily applied for programmed drug delivery. In this review, we have provided the recent literature reports on these modified activities of polymeric micelles. Different methods to characterize polymeric micelles have also been highlighted in the study. Thus polymeric micelles have proved a promising drug delivery carrier to fulfill targeted and localized drug delivery.

\section{Acknowledgments}

None.

\section{Conflicts of interest}

The author declares there is no conflict of interest.

\section{References}

1. Carmen Alvarez Lorenzo, Angel Concheiro. Intelligent Drug Delivery Systems: Polymeric Micelles and Hydrogels. Mini-Reviews in Medicinal Chemistry. 2008;8:1065-1074.

2. Priya Bawa, Viness Pillay, Lisa $\mathrm{C}$ du Toit, et al. Stimuli-responsive polymers and their applications in drug delivery. Biomedical Materials. 2009;4(2):1035-1041.

3. Debashish Roy, Jennifer N Cambre, Brent S Sumerlin. Future perspectives and recent advances in stimuli-responsive materia. Progress in Polymer Science. 2010;3 278-301.

4. Fang Liu, Marek W. Recent advances and challenges in designing stimuliresponsive polymers, Urban. Progress in Polymer Science. 2010;35:3-23.

5. Chi A, Clayton. Intelligent drug-delivery devices based on micro- and nano-technologies. Therapeutic Delivery. 2013;4(1):77-94.

6. SR Croy, GS Kwon. Polymeric Micelles for Drug Delivery. Current Pharmaceutical Design. 2006;12:4669-4684.
7. Carman Alveraz. Light Sensitive Intelligent Drug Delivery System Phytochemistry and photobiology. Wiley Online. 2009;85(4):848-860.

8. Cheng SX, Zhang. Thermo-sensitive polymeric micelles based on poly(Nisopropylacrylamide) as drug carriers. Progress in Polymer Science. 2009;34 (9):893-910.

9. Nostrum V Cornelus. Polymeric micelles for drug delivery. Sci Topics Retri. 2012;12:4669-4684.

10. Wei Xu. Polymeric Micelles, a Promising Drug Delivery System to Enhance Bioavailability of Poorly Water-Soluble Drugs. Journal of Drug Delivery. 2013; 2013:340315.

11. Zaheer Ahmad. Polymeric micelles as drug delivery vehicles. RSC Adv. 2014;4(33):17028-17038.

12. K Yoncheva, P Calleja, M Agueros, et al. Stabilized micelles as delivery vehicles for paclitaxel. International Journal of Pharmaceutics. 2012;436(1):258-264.

13. Zhang J. Host-guest interaction mediated polymeric core-shell assemblies, Versatile nanocarriers for drug delivery. Angew Chem Int Ed Engl. 2009;48:964-68.

14. Jeong YI. Polyion complex micelles composed of all-trans retinoic acid and poly (ethylene glycol)-grafted-chitosan. J Pharm Sci. 2006;95:23482360 .

15. G Ruan, SS Feng. Preparation and characterization of poly(lactic acid)poly(ethylene glycol)-poly(lactic acid) (PLAPEG-PLA) microspheres for controlled release of paclitaxel. Biomaterials. 2003;24(037):5044-2003.

16. N Nishiyama, K Kataoka. Preparation and characterization of sizecontrolled polymeric micelle containing cis dichloro diamine platinum(II) in the core. Journal of Controlled Release. 2001;74(1-3):83-94.

17. A Vila, A Sanchez. Design of biodegradable particles for protein delivery. Journal of Controlled Release. 2002;78(1-3):15-24.

18. YJ Yamanaka, KW Leong. Engineering strategies to enhance nanoparticlemediated oral delivery. Journal of Biomaterials Science Polymer. 2008;19(12):1549-1570.

19. D Rahmat. Thiolated hydroxyethyl cellulose: design and in vitro evaluation of mucoadhesive and permeation enhancing nanoparticles. European Journal of Pharmaceutics and Biopharmaceutics. 2013;83(2):149-155.

20. KMR Srivalli, PK Lakshmi. Design of a novel bilayered gastric mucoadhesive system for localized and unidirectional release of lamotrigine. Saudi Pharmaceutical Journal. 2013;21(1):45-52.

21. LL Fu. Plant lectins: Targeting programmed cell death pathways as antitumor agents. International Journal of Biochemistry and Cell Biology. 2011;43(10):1442-1449.

22. N Yamazaki. Endogenous lectins as targets for drug delivery. Advanced Drug Delivery Reviews. 2000;43(2):225-244.

23. ES Lee, HJ Shin. Poly(L-histidine )PEG block copolymer micelles and $\mathrm{pH}$-induced de-stabilization. Journal of Controlled Release. 2003;90(3):363-374.

24. ES Lee, K Na, YH Bae. Polymeric micelle for tumor $\mathrm{pH}$ and folatemediated targeting. Journal of Controlled Release. 2003;91(1-2):103113

25. Fukushima S, Miyata. A PEG-based biocompatible block catiomer with high buffering capacity for the construction of polyplex micelles showing efficient gene transfer toward primary cells. Chem Med Chem. 2006;1(4):439-444.

26. Chris Oerlemans. Polymeric Micelles in Anticancer Therapy: Targeting, Imaging and Triggered Release. Pharm Res. 2010;27(12):2569-2589.

27. Hamidreza Montazeri. Polymeric Micelles for Drug Delivery. Journal Expert Opinion on Drug Delivery. 2005;3(1):139-162. 
28. Ren-XiZhuo. Thermo-sensitive polymeric micelles based on poly $(N-$ isopropylacrylamide) as drug carriers. Progress in Polymer Science. 2009;34(9):893-910.

29. Rijcken CJ. Triggered de-stabilization of polymeric micelles and vesicles by changing polymers polarity: an attractive tool for drug delivery. $J$ Control Release. 2007;120:131-148.

30. Satturwar P. pH-responsive polymeric micelles of poly(ethylene glycol)b-poly(alkyl(meth) acrylate-co-methacrylic acid): influence of the copolymer composition on self-assembling properties and release of candesartan cilexetil. Eur J Pharm Biopharm. 2007;65:379-87.
31. Salimi. Formulation and Evaluation of Polymeric Micelle Drug Delivery System of Celecoxib To Improve the Oral Absorption. European Journal of Biomedical and Pharmaceutical Sciences. 2015;1(2):372-385.

32. Anayatollah Salimi. Preparation and optimization of polymeric micelles as an oral drug delivery system for deferoxamine mesylate: in vitro and ex vivo studies. Research in Pharmaceutical Sciences. 2019;14(4):293-307. 